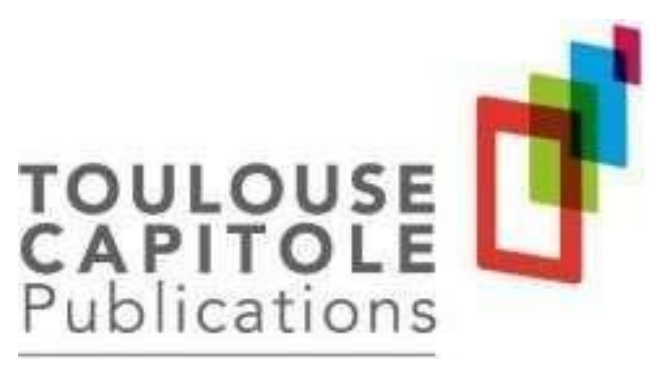

« Toulouse Capitole Publications » est l'archive institutionnelle de l'Université Toulouse 1 Capitole.

Zone agricole et protection de l'environnement : laquelle emporte le PLU?

\author{
PEYEN LOḮC
}

Référence de publication : PEYEN (L.), «Zone agricole et protection de l'environnement : laquelle emporte le PLU ? », Revue Droit et ville (89), 2020, p. 53-71.

Pour toute question sur Toulouse Capitole Publications, contacter portail- publi@ut-capitole.fr 


\section{Zone agricole et protection de l'environnement : laquelle emporte le PLU ?}

Les relations entre activité agricole et protection de l'environnement ne sont guère simples : alors que celle-ci a une influence positive et négative sur celle-là, celle-là à une influence négative et positive sur celle-ci 1. De fait, leurs liens ne sont pas réductibles à l'opposition et au conflit. Dans le contexte du droit de l'urbanisme, qui n'ignore ni les préoccupations environnementales, ni les préoccupations agricoles2, les rapports entre la zone agricole (zone A) et la protection de l'environnement témoignent de cette relation équivoque.

Il convient de préciser que la zone A ne se confond pas avec l'activité agricole : si cette dernière traduit une approche matérielle et désigne une activité (v. par exemple C. rur., art. L. 311-1), la première exprime plutôt une approche territoriale et renvoie à un espace déterminé ou, pour le dire autrement et de façon plus tautologique, que l'on nous pardonne, à une « zone » particulière 3. Elle ne se comprend, en effet, qu'au travers du plan local d'urbanisme (PLU), qui a pour finalité de définir la destination générale des sols au niveau communal (ou intercommunal dans le cadre d'un PLU intercommunal (PLUi)) 4. À côté $\mathrm{du}$ rapport de présentation, du projet d'aménagement et de développement durables (PADD), des orientations d'aménagement et de programmation (OAP) et des annexes (C. urb., art. L. 151-2), le règlement du PLU a une importance capitale : au terme d'une approche fonctionnaliste, il doit « préciser l'affectation des sols selon les usages principaux qui peuvent en être faits ou la nature des activités qui peuvent y être exercées », et ce, en délimitant quatre types de zone (C. urb., art. L. 151-9 et R. 151-17), à savoir les zones urbanisées (zones U), les zones à urbaniser (zones AU), les zones naturelles et forestières (zone N) et, surtout, les zones agricoles (zone A). Mais ce classement ne doit pas être pris pour ce qu'il n'est pas et sa portée ne doit pas être mésestimée. En effet, il ne condamne aucunement les zones à n'accueillir qu'un seul type d'activité : une activité agricole peut tout à fait se dérouler ailleurs qu'en zone A - il suffit de songer à l'agriculture urbaine, par exemple - comme des activités non-agricoles peuvent prendre place en zone A. Il en résulte que si la zone A et l'activité agri- cole se recoupent, elles ne recouvrent pas les mêmes réalités, de sorte qu'étudier les rapports entre zone A et protection de l'environnement n'équivaut pas à traiter de la relation entre activité agricole et environnement, même s'ils ne sont pas sans liens.

En effet, la zone A ne se détache pas de l'activité agricole. Le Code de l'urbanisme ne laisse pas de doute à ce propos car il prévoit que «peuvent être classées en zone agricole les secteurs de la commune, équipés ou non, à protéger en raison du potentiel agronomique, biologique ou économique des terres agricoles » (C. urb., art. R. 151-22). Le classement en zone A suppose donc, outre la volonté de la personne publique à l'origine du PLU, que les parcelles en cause disposent d'un potentiel réel pour l'exercice d'une activité agricole5. Cette vocation signifie donc que la zone A n'a pas pour fonction première de protéger l'environnement.

L'absence de mention d'un potentiel «écologique » des terres à côté d'un éventuel potentiel agronomique, biologique ou économique, contrairement aux zones N (C. urb., art. R. 151-24), en dit long sur ce point. Ce silence distingue clairement la zone A de la «zone de richesses naturelles » (zone NC), qui la précédait avant 2001 dans les plans d'occupation des sols (POS) 6. Ainsi, puisque celle-ci protégeait les zones « en raison notamment de la valeur agricole des terres ou de la richesse du sol ou du sous-sol » (C. urb., ancien art. R. 123-18, I, 2, c), il a été admis qu'une parcelle classée en zone NC puisse être classée soit en zone 
A soit en zone $\mathrm{N}$ selon les cas lors du passage d'un POS à un PLU7. N'ayant plus vocation à protéger la richesse du sol et/ou du sous-sol, dont le régime s'est autonomisé (C. urb., art. R. 151-34), et sans perdre de vue le fait qu'il existait auparavant des zones ND, dont l'intérêt environnemental était bien plus grand (ancien art. R. 123-18, I, 2, d) et auxquelles ont succédé les actuelles zones N, il est permis d'affirmer que le champ de la zone A est beaucoup plus restreint que celui de l'ancienne zone NC. La raison d'être de la zone A est donc résolument plus « agricole » qu'environnementale. Heureusement, cela n'empêche pas qu'elle noue des relations particulières avec la protection de l'environnement.

Et pour cause : la préservation des terres agricoles n'est pas incompatible per se avec la protection de l'environnement. Le régime juridique de la zone A le montre clairement : contribuant à lutter contre l'extension de l'urbanisation, entre autres choses, le classement en zone A a un effet bénéfique sur l'environnement (I). Néanmoins, parce que la zone A s'insère dans un environnement plus global, les exigences tenant à la protection de ce dernier sont à l'origine de certaines restrictions dans la zone A, laquelle ne peut faire fi de son contexte (II).

\section{I. $\quad$ LES EFFETS DE LA ZONE A SUR LA PROTECTION DE L’ENVIRONNEMENT}

De par son régime juridique, qui se distingue fondamentalement de ceux des zones $U$ et des zones AU, la zone A se présente comme un outil réellement intéressant de protection de l'environnement (A), même si sa contribution n'est pas sans limites (B).

A.

\section{LA CONTRIBUTION CERTAINE DE LA ZONE A À LA PROTECTION DE L'ENVIRONNEMENT}

Le classement en zone A a des conséquences extrêmement importantes car, contrairement aux zones U et AU, elle soumet les parcelles classées à un principe d'inconstructibilité. Un tel classement ferme donc les parcelles qui en sont l'objet à l'urbanisation et, pour cette raison, il contribue à lutter contre l'urbanisation toujours plus rampante. Cela ne saurait étonner dans la mesure où sa raison d'être est de protéger les secteurs de la commune « en raison du potentiel agronomique, biologique ou économique des terres agricoles » (C. urb., art. R. 151-22).

Il s'agit là de l'apport le plus significatif de la zone A à la protection de l'environnement : elle participe à la protection des espaces « naturels », entendus comme des espaces non-urbanisés8. Réduisant le grignotage des « campagnes », en particulier lorsqu'elle se retrouve dans les espaces périurbains 9, elle encourage la construction de la ville sur elle-même, c'est-à-dire la densification plus que l'étalement, et impose de trouver des solutions innovantes pour adapter les espaces urbains aux défis auxquels ils sont confrontés, comme la pression démographique.

Cette propriété du régime juridique de la zone A la rapproche de la zone $\mathrm{N}$, dont la vocation environnementale est bien plus manifeste. En effet, même si chacune de ces zones a son objet propre et dispose à ce titre de spécificités, elles font l'objet d'un traitement conjoint dans le règlement du PLU qui les distingue clairement des zones U et AU. Il en ressort une approche binaire qui oppose d'un côté les zones constructibles et de l'autre celles qui ne le sont pas 10. Le code de l'urbanisme en témoigne : à propos de l'affectation des sols et de la destination des constructions, il traite séparément des « zones naturelles, agricoles ou forestières » (art. L. 151-11 et s.) et des « zones urbaines ou à urbaniser » (art. L. 151-14 et s.). Cette identité de traitement avec la zone $\mathrm{N}$ et leur soumission commune au principe d'inconstructibilité ne sont rien d'autre que l'expression du rôle environnemental majeur qui leur est 
assigné : elles limitent l'urbanisation. Il convient cependant de préciser que le classement en zone A n'est pas incompatible avec l'urbanisation existante des parcelles : non seulement il peut intervenir alors même que ces dernières comportent des constructions11, mais en plus, il n'implique pas leur démolition. Limitant malgré tout les possibilités d'urbanisation future de la parcelle, ce qui n'est pas rien, il est tout à fait possible de considérer qu'il contribue à freiner l'urbanisation; dès lors, même le classement en zone A d'une parcelle accueillant des constructions peut limiter l'étalement urbain (sur la teneur de ce rôle, v. infra, partie II, B).

La consistance de ce rôle n'est pas dissociable, au demeurant, de la valeur et de la portée du règlement du PLU. Par le haut, il est limité par les règles posées par le législateur, en particulier celle de l'inconstructibilité de principe ; concrètement, cela signifie que les auteurs du PLU ne sauraient y déroger et que toute zone $\mathrm{A}$ est concernée par cette règle. Classer une parcelle en zone A revient donc à accepter le régime juridique qui y est associé et à l'appliquer. Par le bas, le règlement doit préciser les règles compte tenu des circonstances locales et, surtout, il s'impose dans un rapport de conformité - le plus exigeant en droit de l'urbanisme ! - aux travaux, constructions, aménagements, plantations, affouillements ou exhaussements des sols et à certaines installations classées (C. urb., art. L. 152-1) 12. Autrement dit, il s'impose sans qu'il soit possible pour les administrés de disposer d'une marge de manœuvre à son égard. Ceci explique pourquoi le classement d'une parcelle en zone A peut affecter sa valeur et être si problématique pour les propriétaires en même temps qu'elle contribue à la protection de l'environnement. Mais en vérité, comme tout principe, le principe d'inconstructibilité en zone A n'est pas absolu, et ses exceptions sont notables (C. urb., art. L. 151-11 et s.). L'une d'elles mérite d'ailleurs une attention particulière en ce sens qu'elle contribue, d'une autre façon, à la protection de l'environnement. Il s'agit de l'une des premières exceptions prévues par le code - le positionnement en dit long -, et elle permet d'" autoriser les constructions et installations nécessaires à des équipements collectifs dès lors qu'elles ne sont pas incompatibles avec l'exercice d'une activité agricole, pastorale ou forestière du terrain sur lequel elles sont implantées et qu'elles ne portent pas atteinte à la sauvegarde des espaces naturels et des paysages » (C. urb., art. L. 151-11, I, $1^{\circ}$ ). Cette exception, qui indique qu'un intérêt général (« équipement collectif »13) peut venir adou- cir le principe d'inconstructibilité, permet l'implantation sous conditions en zone A d'installations de production d'énergies renouvelables, qu'il s'agisse d'éoliennes 14 ou d'installations d'énergie solaire15 par exemple. Dans cette lignée, le Conseil d'État a récemment rappelé qu'il était possible pour les constructions et installations à usage agricole de servir à d'autres activités, notamment de production d'énergie, sans pour autant perdre leur caractère agricole, dès lors que ces activités annexes ne remettent pas en cause la destination agricole avérée des constructions et installations en cause 16. Cette exception est marquante en ce sens qu'elle donne une nouvelle dimension à la contribution de la zone A à la protection de l'environnement : son œuvre n'est pas seulement passive et négative du fait de sa seule existence et des limitations à l'urbanisation qu'elle emporte, elle est aussi active et positive dans la mesure où elle rend possible la production d'énergies «vertes ». Au fond, il s'agit de rentabiliser l'espace agricole d'un point de vue environnemental sans pour autant le dénaturer.

La contribution de la zone A à la protection de l'environnement est donc réelle, même si, une fois le plan des principes dépassé, cet apport doit être relativisé.

\section{B. LA CONTRIBUTION LIMITÉE DE LA ZONE A À LA PROTECTION DE L'ENVIRONNEMENT}

Cela a été dit : en leur permettant d'avoir une certaine maîtrise sur le foncier agricole, la zone A constitue un outil à disposition des collectivités publiques à l'origine du PLU pour protéger les terres agricoles et 
lutter contre l'urbanisation. À ce titre, le classement de telle ou telle parcelle relève de la volonté de ces collectivités. Or, celles-ci devant souvent arbitrer entre des intérêts et des enjeux multiples, la protection de l'environnement, par le truchement de la protection des terres agricoles, n'est assurée que si elle prime la volonté d'urbaniser. En d'autres termes, si le classement en zone A présente un intérêt certain pour la conservation de l'environnement, ce n'est qu'à la condition que la personne publique ait pu résister à la pression des forces sociales locales et/ ou qu'elle ait voulu qu'une telle zone soit instituée. Sur ce point, si les auteurs du PLU se confrontent à certaines limites, ils disposent tout de même d'une certaine marge de manœuvre.

Les limites se retrouvent essentiellement dans les documents supérieurs qui s'imposent au PLU (v. notamment C. urb., art. L. 131-4), comme le Schéma de cohérence territoriale (SCoT). Celui-ci doit dans son rapport de présentation, analyser la consommation d'espaces naturels, agricoles et forestiers au cours des dix années précédant l'arrêt du projet de schéma et justifier les objectifs chiffrés de limitation de cette consommation compris dans le document d'orientation et d'objectifs (DOO) (C. urb., art. L. 141-3, al. 4) 17. Ce dernier, qui doit notamment déterminer « les orientations générales de l'organisation de l'espace et les grands équilibres entre les espaces urbains et à urbaniser et les espaces ruraux, naturels, agricoles et forestier » ainsi que « les conditions d'un développement équilibré dans l'espace rural entre l'habitat, l'activité économique et artisanale, et la préservation des sites naturels, agricoles et forestiers » (C. urb., art. L. 141- $5,1^{\circ}$ et $3^{\circ}$ ), doit aussi arrêter objectifs chiffrés de consommation économe de l'espace et de lutte contre l'étalement urbain par secteur géographique et décrire pour chacun d'eux les enjeux qui leur sont propres (C. urb., art. L. 141-6) 18 et identifier les espaces et sites naturels, agricoles, forestiers ou urbains à protéger en définissant leur localisation ou leur délimitation (C. urb., art. L. 141-10). Or, les PLU doivent être compatibles avec le DOO (C. urb., art. L. 131-4 et L. 142-1), ce qui restreint la marge de manœuvre des auteurs du PLU, même si le juge apprécie cette compatibilité de façon globale 19. Il en est de même, s'agissant, outre-mer, de la Guadeloupe, la Guyane, la Martinique, Mayotte et La Réunion, où les enjeux agricoles sont considérables, du Schéma d'aménagement régional (SAR) (CGCT, art. L. 4433-7 et s.). Ce dernier, qui s'impose dans un rapport de compatibilité aux SCoT (C. urb., art. L. 131-1 $4^{\circ}$ ), et, en son absence, aux PLU (C. urb., art. L. 131-7), peut imposer aux documents de planification qui lui sont inférieurs de classer de façon appropriée des parcelles pour la préservation de leur vocation agricole, limitant ipso facto la marge de manœuvre des collectivités 20. Ces contraintes, si elles sont réelles, n'empêchent pas qu'il y a à leur base une volonté publique, laquelle doit nécessairement prendre en compte des intérêts multiples, souvent contradictoires, ce qui lui impose de faire des choix et, partant, des compromis 21. Ce subjectivisme, si regrettable puisse-t-il être, est néanmoins indissociable de la norme urbanistique qui est nécessairement territorialisée. Autrement dit, la protection de l'environnement par la zone A n'est possible que tant que les décisionnaires ne choisissent pas ou ne sont pas contraints de la sacrifier pour faire valoir d'autres intérêts, ce qui la place, dès lors, dans une situation pour le moins délicate22. Cela dit, il y a des raisons de rester optimiste et croire en la bonne volonté des personnes publiques : le juge administratif a pu admettre la légalité du classement en zone A de parcelles car elle traduit la « volonté des auteurs du PLU de préserver l'avenir de l'agriculture et de lutter contre l'étalement urbain »23. Mais il faut tout de même rester mesuré. La contribution de la zone A à la protection de l'environnement s'arrête à l'objet du classement : il ne sert qu'à protéger un espace et sa vocation agricole et n'a pas, de ce fait, d'incidence sur les caractéristiques des activités qui pourront y prendre place. Déjà, parce qu'il encourage la réalisation d'activités agricoles, bien connues pour leurs effets néfastes sur l'environnement, il concourt à la détérioration de la qualité de l'environnement. Il ne s'oppose pas par principe à ce que des installations classées pour la protection de l'environnement agricoles soient implantées sur les parcelles qu'il concerne 24, pas plus qu'il n'interdit la pratique d'une agriculture 
intensive. Il ne peut non plus imposer que les activités sur la parcelle se déroulent dans les conditions les plus favorables à la protection de l'environnement, qu'il s'agisse d'une agriculture biologique ou d'une alimentation animale ne participant pas à la déforestation importée : "la règle d'urbanisme peut se préoccuper ici ou là de préserver l'activité agricole, mais elle n'a pas pour vocation d'indiquer quel type de culture on peut pratiquer ici ou là » 25. En fait, comme la réglementation de l'urbanisme en général (C. urb., art. L. 101-3), le PLU n'a pas vocation à règlementer l'activité agricole et « ne doit pas fixer de règles en ce qui concerne la production agricole $» 26$, qui relève davantage du Code rural et de la pêche maritime en vertu du principe d'indépendance des législations 27. Tout au plus la volonté de parvenir à une agriculture durable peut-elle figurer dans le PADD du PLU : définissant notamment les orientations générales des politiques de protection des espaces agricoles (C. urb., art. L. 151-5), ce dernier, même s'il n'est pas opposable aux autorisations d'urbanisme28, a tout de même une incidence sur le règlement, qui doit fixer les règles générales et les servitudes d'utilisation des sols en cohérence avec le PADD (C. urb., art. L. 151-8) 29. Cette remarque sur la finalité du droit de l'urbanisme ne vaut bien évidemment pas que pour la zone A, mais elle a son importance lorsqu'il est question de mesurer la contribution de cette dernière à la protection de l'environnement.

Enfin, la contribution de la zone A à la protection, voire à l'amélioration, de l'environnement est limitée car, si elle est intrinsèquement liée à l'activité agricole, cette dernière s'autonomise volontiers, atténuant quelque peu l'intérêt d'un classement la zone A. Ce constat vaut surtout pour l'agriculture urbaine qui, permettant de ramener la nature en ville, contribuerait à l'amélioration de l'environnement urbain (amélioration du cadre de vie, constitution d'îlots de fraîcheur, amélioration de la qualité paysagère des lieux, etc.). Or, il n'est pas certain que le classement en zone A de parcelles situées en milieu urbain ne relève pas de l'erreur manifeste d'appréciation. En effet, pour apprécier la légalité du classement en zone A, le juge administratif peut vérifier que la par- celle concernée n'est pas enclavée « au milieu de parcelles qui seraient toutes constructibles » ou « au milieu de parcelles classées en zone autorisant une urbanisation » 30. Il vérifie encore parfois que la parcelle qui jouxte une zone $\mathrm{AU}$ « se rattache à une vaste zone de culture à vocation agricole » 31. La menace ne semble cependant pas miner la motivation de certaines collectivités qui font parfois preuve d'audace en franchissant ce pas32. Mais l'intérêt de recourir à la zone A n'en demeure pas moins limité dans ce cadre puisque le Code de l'urbanisme permet au règlement, pour parvenir aux mêmes fins, de « localiser, dans les zones urbaines, les terrains cultivés et les espaces non bâtis nécessaires au maintien des continuités écologiques à protéger et inconstructibles quels que soient les équipements qui, le cas échéant, les desservent » (art. L. 151-23, al. 2), et ce, quelle que soit la valeur agronomique des sols33. Dès lors, dans de telles circonstances, le classement en zone A peu paraître assez hasardeux.

Enfin, il convient de préciser que les exceptions au principe d'inconstructibilité en zone A appellent également quelques remarques car elles permettent une urbanisation encadrée des zones A. Néanmoins, outre le fait que le principe reste l'inconstructibilité, ces exceptions restent conditionnées par certaines considérations environnementales (sur la teneur de ces conditions, v. infra, partie II, B).

Il ressort de l'ensemble de ces éléments que la zone A présente un intérêt réel pour protéger l'environnement, même s'il importe de relativiser cet apport tant ces limites sont conséquentes. Sans doute faut-il se réjouir qu'il contribue en partie à une telle œuvre, là où la zone $U$ et la zone AU peuvent laisser plus songeur. En tout état de cause, les relations entre zone A et protection de l'environnement sont duales : si la zone A contribue à la protection de l'environnement, les exigences tenant à la protection de l'environnement produisent également des effets sur la zone A. 


\section{LES EFFETS DE LA PROTECTION DE L'ENVIRONNEMENT SUR LA ZONE A}

Il paraît assez évident que la zone A ne peut se couper de son contexte. Du point de vue de son environnement normatif, le PLU - et ses éléments constitutifs, donc - se doit de respecter toutes les normes qui lui sont supérieures et notamment les principes et objectifs des articles L. 101-1 à L. 101-3 (C. urb., art. L. 151-1 et L. 151-8) parmi lesquels figurent l'objectif d'équilibre entre les espaces urbanisés et les espaces non-urbanisés, naturels ou agricoles, au risque sinon d'une annulation par le juge administratif34. Il en va naturellement de même des objectifs et principes contenus dans la Charte de l'environnement, dont le rang constitutionnel permet leur pénétration dans le droit de l'urbanisme 35, au point qu'il n'est pas impossible d'affirmer aujourd'hui que ce der- nier poursuit aujourd'hui un objectif général de développement durable 36. La zone A, par voie de conséquence, ne peut être hermétique aux considérations environnementales. Cette exigence de droit rejoint une exigence de fait : la zone A appartient au monde. Plus exactement, elle n'est pas un espace isolé de son environnement et doit composer avec les exigences de la protection de ce dernier, qui lui imposent de s'insérer au mieux dans le réel. Ceci explique pourquoi la protection de l'environnement est à l'origine, dans la zone $\mathrm{A}$, de contraintes générales, c'est-à-dire qui ne lui sont pas propres (A), et spécifiques, qui la concernent spécialement (B).

\section{A. LES CONTRAINTES GÉNÉRALES DE LA PROTECTION DE L'ENVIRONNEMENT SUR} LA ZONE A

Le classement en zone A d'une parcelle ne se fait pas dans l'indifférence de paramètres environnementaux. Ainsi, il a été considéré comme possible, pour le classement d'une parcelle en zone $\mathrm{NC}$, de prendre en compte la « richesse naturelle des lieux » 37 ou encore «des considérations liées à la protection contre les risques d'incendie, dès lors qu'un classement ainsi motivé concourt à la protection des richesses naturelles qui caractérisent les parcelles en cause et le secteur dans lequel elles s'insèrent » 38. L'intégration de ces paramètres environnementaux se justifie aussi parce que le PLU s'insère dans une hiérarchie des normes urbanistiques qui lui impose le respect de plusieurs documents (C. urb., art. L. 1314 et s.) 39. Cette situation a un effet direct sur le classement en zone A. La charte d'un parc naturel régional, qui s'impose au PLU dans un rapport de compatibilité en l'absence de SCoT (C. urb., art. L. 131-7 et L. 131-1), est par exemple susceptible de remettre en question le classement d'une parcelle en zone A40. Bien sûr, le propos vaut aussi pour les autres normes à dimension environnementale et les énumérer ici ne présenterait que peu d'intérêt 41 . Il est en revanche utile d'apprécier l'étendue des contraintes environnementales sur la zone $\mathrm{A}$, qui vont au-delà de la seule application de la règle hiérarchique.

Et pour cause : le classement d'une parcelle en zone A ne la détache pas de son environnement et, pour cette raison, elle se doit de s'intégrer à lui. Ainsi le règlement du PLU peut-il imposer en zone $\mathrm{A}$, comme dans ses autres zones, des règles particulières en considération de l'environnement (C. urb., art. L. 15117 et s.). Celles-ci sont diverses par leur objet et leur teneur car elles peuvent : porter sur l'implantation et l'aspect extérieur des constructions « afin de contribuer à la qualité architecturale, urbaine et paysagère, à la mise en valeur du patri- moine et à l'insertion des constructions dans le milieu environnant » (C. urb., art. L. 151-18 ; v. aussi les art. R. 151-39, R. 151-41 et R. 151-42) ; comporter des prescriptions spéciales pour protéger des éléments de paysage (art. L. 151-19) ainsi que « les sites et secteurs à protéger pour des motifs d'ordre écologique, notamment pour la préservation, le maintien ou la remise en état des continuités écologiques » (C. urb., art. L. 151-23, al. 1 ; v. aussi R. 151-34 et R. 141-43) ou des prescriptions en 
matière de performances énergétiques et environnementales des bâtiments (C. urb., art. L. 151-21 et R. 151-42). Le règlement peut encore faire apparaître « les secteurs où les nécessités du fonctionnement des services publics, de l'hygiène, de la protection contre les nuisances et de la préservation des ressources naturelles ou l'existence de risques naturels, de risques miniers ou de risques technologiques justifient que soient interdites les constructions et installations de toute nature, permanentes ou non, les plantations, dépôts, affouillements, forages et exhaussements des sols » ou les espaces boisés classés de l'article L. 113-1 (C. urb., art. R. 151-31). Cet ensemble constitue une base et n'est pas figé puisque " les règles générales peuvent être assorties de règles alternatives qui en permettent une application circonstanciée à des conditions locales particulières » (C. urb., art. R. 151-13).

Par ailleurs, un durcissement des règles applicables en zone A est envisageable en présence d'un espace protégé. Le Code de l'urbanisme, pour ne parler que de lui, dans le chapitre 3 du titre 1er du Livre 1er de sa Partie législative, intitulé « Espaces protégés » (C. urb., art. L. 113-1 et s.), institue un certain nombre de dispositifs de protection d'espaces particuliers, qui permettent le classement de certaines zones en considération de paramètres particuliers. Lorsque ces zones se trouvent en zone A, des exigences particulières viennent enrichir son régime juridique.

Il en va ainsi, d'abord, des espaces boisés (C. urb., art. L. 113-1 et s. ; art. R. 113-1 et s.), dont l'existence " n'est pas incompatible avec le classement de parcelles en zone agricole par le plan local d'urbanisme » 42. Ce statut interdit tout changement d'affectation ou tout mode d'occupation du sol de nature à compromettre la conservation, la protection ou la création des boisements, comme les opérations de défrichement, les coupes ou abattages d'arbres isolés, de haies ou réseaux de haies et de plantations d'alignement (C. urb., art. L. 113-2).

Il en est de même, ensuite, des espaces naturels sensibles qui permettent aux départements de préserver la qualité des sites, des paysages, des milieux naturels et des champs naturels d'expansion des crues et d'assurer la sauvegarde des habitats naturels (C. urb., art. L. 113-8 et s. ; art. R. 113-15 et s.). Un tel classement rend possible la création de zones de préemption 43 (C. urb., art. L. 113-14), y compris en zone A 44.

Cela concerne aussi, depuis 201645, les espaces de continuités écologiques que les PLU peuvent identifier, et qui renvoient aux éléments des trames verte et bleue (C. env., art. L. 371-1) qui sont nécessaires à la préservation ou à la remise en bon état des continuités écologiques (C. urb., art. L. 113-29 et s.). L'objectif de conciliation entre protection de l'environnement et activités humaines conduit à ce que cette protection soit assurée « en tenant compte des activités humaines, notamment agricoles » (C. urb., art. L. 113-30), sans davantage de précisions. La mention des activités agricoles laisse entendre que la préservation de ces dernières ne doit pas être sacrifiée sur l'autel de la protection des continuités écologiques 46.

Enfin, cela vaut également pour les espaces agricoles et naturels périurbains (C. urb., art. L. 113-15 et s. ; art. R. 113-19 et s.), qui sont créés pour protéger les espaces agricoles et naturels périurbains de l'urbanisation 47. Ici, il est rendu possible aux départements et à certains établissements publics de délimiter des périmètres d'intervention pour la protection et la mise en valeur des espaces agricoles et naturels périurbains, et ce, uniquement dans les zones $\mathrm{N}$ et A (pour le détail : C. urb., L. 113-17). À l'intérieur de ces périmètres, se mettront en œuvre des programmes d'action qui « précise[nt] les aménagements et les orientations de gestion destinés à favoriser l'exploitation agricole, la gestion forestière, la préservation et la valorisation des espaces naturels et des paysages au sein du périmètre 
d'intervention » (C. urb., art. L. 113-21). Ce dispositif, outre le fait de contribuer à lutter contre l'étalement urbain, peut conduire à l'acquisition de biens par le biais d'une procédure d'expropriation (C. urb., art. L. 113-24) ou de préemption (C. urb., art. L. 113-25).

Il convient toutefois de préciser que l'ensemble de ces contraintes relèvent plus de l'indicatif que de l'impératif, de la faculté plus que de l'obligation 48. Par conséquent, les effets des enjeux environnementaux sur la zone A relèvent essentiellement, dans leur teneur et leur étendue, de la volonté d'entités multiples, qu'il s'agisse de collectivités ou d'établissements publics, au point qu'il est possible de s'interroger sur la réelle portée pratique de ses possibilités théoriques de protection de l'environnement. En ceci : toutes ces entités sont d'une façon ou d'une autre confrontées à une multitude d'intérêts et il est nécessaire que celles-ci conservent une marge de manœuvre, sans quoi ce serait tout le droit de l'urbanisme et son architecture qu'il faudrait remettre en cause49. Mais, dans la mesure où l'environnement ne saurait se protéger tout seul, il est possible de s'interroger sur les modalités de sa protection, notamment en zone A, et, partant, se demander s'il ne serait pas mieux de raffermir l'ensemble de ces dispositions, s'agissant surtout des espaces fragiles, pour obliger les PLU à intégrer de façon convenable les enjeux environnementaux, et ce, sans que cela n'empêche les arbitrages qui s'imposeraient en fonction des circonstances locales. En d'autres termes, il s'agirait de consolider les exigences existantes en la matière tout en ménageant aux autorités locales une certaine marge de manœuvre pour opérer les équilibres les meilleurs, pour peu que l'expression ait réellement un sens - tout étant question de point de vue.

Cela étant, il existe dans les zones A, à côté de ces contraintes générales, des contraintes spécifiques tenant à la protection de l'environnement.

\section{B. LES CONTRAINTES SPÉCIFIQUES DE LA PROTECTION DE L'ENVIRONNEMENT SUR LA ZONE A}

La zone A connaît des contraintes relatives à la protection de l'environne- ment qui lui sont propres, quoique partagées avec la zone $\mathrm{N}$ au vu du traitement quasi simultané dont elles font l'objet. Ces contraintes se manifestent surtout à propos des exceptions au principe d'inconstructibilité qui existent, et dont la réalisation est globalement conditionnée par certains paramètres environnementaux. Ces exceptions sont synthétisées dans l'article R. 151-23 du Code de l'urbanisme : il y a d'un côté, les constructions et installations nécessaires à l'exploitation agricole50 ou au stockage et à l'entretien de matériel agricole par les coopératives d'utilisation de matériel agricole agréées, et, de l'autre, celles fixées par le législateur qui intègrent véritablement des enjeux environnementaux.

En premier lieu, les constructions et installations nécessaires à des équipements collectifs (C. urb., art. L. 151-11, $\mathrm{I}, 1^{\circ}$ ) ne peuvent être autorisées que si, en plus de ne pas être incompatibles avec l'exercice d'une activité agricole, ils ne portent pas atteinte à la sauvegarde des espaces naturels et des paysages. En deuxième lieu, le changement de destination de bâtiments n'est possible que si ces derniers ont été désignés par le règlement et uniquement si ce change- ment ne compromet pas l'activité agricole ou la qualité paysagère du site. Dans la zone $\mathrm{A}$, un tel changement est soumis à l'avis conforme de la commission départementale de la préservation des espaces naturels agricoles et forestiers (CDPENAF) (C. urb., art. L. 151-11, I, $2^{\circ}$ et R. 151-35).

En troisième lieu, les constructions et installations nécessaires à la transformation, au conditionnement et à la commercialisation des produits agricoles ne peuvent être autorisées, quant à elles, que lorsque ces 
activités constituent le prolongement de l'acte de production, et uniquement si elles ne sont pas incompatibles avec l'exercice d'une activité agricole, pastorale ou forestière sur le terrain sur lequel elles sont implantées et si elles ne portent pas atteinte à la sauvegarde des espaces naturels et des paysages. Dans ce cas, l'autorisation d'urbanisme est soumise pour avis à la CDPENAF (art. L. 151-11, II).

En quatrième lieu, la réalisation d'extensions et d'annexes des bâtiments d'habitation existants n'est permise que si elle ne compromet pas l'activité agricole ou la qualité paysagère du site et, sous réserve, là encore, de l'avis de la CDPENAF. Dans ces cas, le règlement doit préciser les éléments " permet- tant d'assurer leur insertion dans l'environnement et leur compatibilité avec le maintien du caractère naturel, agricole ou forestier de la zone » (C. urb., art. L. 151-12) 51.

En cinquième lieu, le règlement peut délimiter « à titre exceptionnel » certains secteurs de taille et de capacité d'accueil limitées dans lesquels peuvent être autorisés des constructions, des aires d'accueil et des terrains familiaux locatifs destinés à l'habitat des gens du voyage ou des résidences démontables constituant l'habitat permanent de leurs utilisateurs. Dans ces cas, il doit notamment préciser les éléments permettant d'assurer leur insertion dans l'environnement et leur compatibilité avec le maintien du caractère naturel, agricole ou forestier de la zone. La délimitation de ces secteurs exige également l'avis de la CDPENAF (C. urb., art. L. 151-13).

Il appert que ces exceptions sont systématiquement conditionnées par certains paramètres environnementaux et cela pourrait, à juste titre, susciter un certain enthousiasme. Néanmoins, plusieurs remarques doivent être faites pour que cet état du droit puisse être apprécié à leur juste valeur.

Premièrement, le nombre de ces exceptions permet de relativiser la portée du principe d'inconstructibilité et, ce faisant, la contribution de la zone A à la lutte contre l'urbanisation. Même si le passage de la zone $\mathrm{NC}$ à la zone $\mathrm{A}$ a pu renforcer initialement le principe d'inconstructibilité52, le nombre d'exceptions à ce dernier n'a cessé de croître au fil des ans, au point de l'affaiblir de façon significative. Que l'on en juge. Les exceptions relatives aux activités nécessaires à l'activité agricole et aux équipements collectifs sont les plus anciennes, mais vinrent se rajouter celle relative au changement de destination des bâtiments en 200353, celle ayant trait aux secteurs «permissifs » de taille et de capacité d'accueil limitées en 201054 , celle portant sur les extensions des bâtiments d'habitation en 201455, celle concernant leurs annexes en 201556, celle renvoyant aux coopératives d'utilisation de matériel agricole agrées en 201557 et, enfin, celle correspondant aux activités constituant le prolongement de l'acte de production en 201858. Au fond, l'histoire de ces exceptions est celle de leur multiplication et, chemin faisant, celle du recul du principe d'inconstructibilité. Certes, au fur et à mesure les conditions se sont renforcées et précisées, mais, même de ce point de vue, il est possible d'être dubitatif.

Deuxièmement, en effet, le conditionnement environnemental des exceptions n'apparaît pas comme une garantie effective de la protection de l'environnement en zone A. Sur son champ d'application d'abord, il est manifeste qu'il ne concerne pas toutes les exceptions, comme celle relative aux activités nécessaires à l'activité agricole ou impliquant les coopératives d'utilisation de matériel agricole. Sur sa teneur, très concrètement, ce critère est loin d'être le plus décisif puisque l'attention des requérants, et donc des juges, se focalise quasi systématiquement sur la non-incompatibilité de l'objet de l'exception en cause avec l'exercice d'une activité agricole plus que sur son effet sur les milieux et les paysages. Cela s'explique sans doute par le caractère cumulatif de ces critères : si le premier fait défaut, le juge estime qu'il n'est pas nécessaire de vérifier le second 59. C'est peut-être ce qui justifie aussi que le Conseil d'État se soit 
attaché à expliciter les modalités d'appréciation de la première condition, sans se risquer à l'obiter dictum à propos de la seconde $60 . .$. alors que l'on connaît la Haute juridiction administrative plus courageuse par ailleurs. Bien heureusement, il est possible de tempérer la critique : le juge veille tout de même en censurant les cas où rien n'est fait pour que les constructions s'intègrent à leur environnement 61. Aussi, il serait possible d'affirmer que l'appréciation d'une atteinte à un paysage est subjective et qu'à partir du moment où des éoliennes ou d'autres constructions massives sont permises, c'est que le critère fait l'objet d'une appréciation souple. Cela ne serait pas sans doute pas totalement faux, mais ce serait oublier que, dans bien des cas, ces installations requièrent des évaluations environnementales qui permettent d'apprécier leur insertion dans leur environnement, laissant alors au juge la possibilité de vérifier au terme d'un contrôle entier le respect du critère environnemental62. En d'autres termes, il ne faut pas négliger les ponts entre les différents dispositifs juridiques qui permettent, au final, une prise en compte de l'environnement dans la zone A. Troisièmement, l'effet combiné de ces exceptions ne peut non plus être négligé : et si, après un changement de destination d'un bâtiment, le propriétaire souhaitait réaliser une extension ou une annexe ? Il n'est pas certain qu'en l'état actuel du droit cette urbanisation insidieuse ne puisse se faire.

Enfin, de façon plus globale, les contraintes relatives à la protection de l'environnement spécifiques à la zone A se manifestent également, au-delà des exceptions au principe d'inconstructibilité, en zone littorale ou en zone de montagne, où des aménagements juridiques spéciaux peuvent intervenir dans un sens ou l'autre 63. Il en est de même pour les autres espaces protégés en général, qu'il s'agisse des zones humides 64 ou des zones Natura 200065, entre autres 66.

La zone A n'ignore donc pas les exigences tenant à la protection de l'environnement, et qui sont à l'origine de contraintes générales et spécifiques. Au fond, il est même possible de soutenir que ces contraintes ne sont en réalité que la contrepartie des effets sur l'environnement qu'est susceptible d'avoir la zone A, comme si les unes ne pouvaient aller sans les autres.

\section{CONCLUSION}

Les relations entre zone A et protection de l'environnement ne sont en définitive pas simples : ne se réduisant pas à l'opposition et au conflit, elles sont au contraire d'une grande richesse. S'il est permis de se satisfaire de la contribution indéniable de la zone A à la protection de l'environnement, les zones de faiblesse identifiées ne peuvent que laisser place au doute, à l'incertitude, voire à l'amertume. Sans doute s'agit-il là d'une conséquence du fait qu'elle n'est pas autre chose qu'un « espace partiellement affecté à la protection de la nature $» 67$. Là où la zone $\mathrm{A}$ peut se présenter comme un rempart face à l'urbanisation, il est possible d'y déceler des zones de fragilité et d'ouverture qui, ne cessant de s'agrandir, ne peuvent qu'interpeller sur la concrétisation de la conciliation entre la protection de l'environnement et les activités humaines. Là où le Code de l'urbanisme prophétise un équilibre improbable, au risque de se perdre 68, il se présente davantage comme un code de l'urbanisation, qui indique la marche à suivre pour construire en détruisant peu... mais tout de même. C'est, au final, une assourdissante inquiétude qui surgit : que le droit de l'urbanisme ne soit pas en mesure de protéger véritablement l'environnement, eu égard à son ambition de conciliation, est une chose, mais qu'il ne parvienne pas, en amont, à protéger l'essence de la zone A, qui est l'une des clés d'avenir de la protection des milieux naturels face à l'urbanisation, c'en est une autre. 


\section{Notes de bas de page}

1. V. surtout M. Prieur (en collab.), Droit de l'environnement, 8e éd., Dalloz, 2019, p. 1139 et s.

2. Y. Jégouzo et P. Planchet, « Agriculture », in Dictionnaire du droit de l'urbanisme. Diction- naire pratique, Y. Jégouzo et N. Foulquier (dir.), 3e éd., Le Moniteur, 2019, p. 48-55.

3. Sous l'angle de la distinction entre territoire rural et espace agricole : F. Barbier, « Le terri- toire rural dans les documents d'urbanisme : quelles difficultés pour l'exercice des activités agricoles ? », Dr. rur., $\mathrm{n}^{\circ} 399$, janvier 2012, p. 32-35.

4. Il est à noter que le principe d'indépendance des législations limite la portée de l'article

L. 311-1 du code rural et de la pêche maritime : ce dernier ne peut servir de fondement exclusif aux décisions prises dans un domaine autre, comme en matière d'urbanisme ou de fiscalité, même s'il peut les inspirer.

5. Le juge administratif exerce sur le classement un contrôle de l'erreur manifeste d'apprécia- tion. Pour ce faire, il apprécie le « potentiel » des terres au regard de l'usage agricole qu'il est possible d'en faire. En ce sens, le juge administratif estime que, au moment de leur classement, les parcelles concernées n'ont pas à être le support d'une activité agricole ; simplement, elles doivent disposer d'un potentiel particulier pour une telle activité. Ainsi, peuvent être classées en zone A des parcelles qui étaient «insérées dans un secteur à dominante rurale et de caractère agricole », et ce, quelle que soit leur valeur agricole (CAA Bordeaux, 30 décembre 2005, Commune d'Aslonnes, nº 02BX02119 : Constr.-Urb. 2006, $\mathrm{n}^{\circ}$ 4, p. 25 , note Rousseau). Cette formulation maladroite renvoie en réalité à une approche contextuelle pour apprécier le potentiel agricole de terres. Cependant, cette approche n'est pas exclusive et certains juges peuvent retenir une approche plus matérielle fondée sur l'usage réel ou potentiel des terres (pour un exemple récent : CAA Lyon, 2 avril 2020, n $^{\circ}$ 19LY00811). Le Conseil d'État a quant à lui pu affirmer qu'une parcelle qui supportait déjà une construction, mais qui ne faisait pas l'objet d'une exploitation agricole et qui ne présentait pas « un potentiel particulier pour un tel usage » ne saurait être classée en zone agricole (CE, 4 mars 2016, Commune de Martigues, $\left.\mathrm{n}^{\circ} 384795\right)$.

6. Décret $\mathrm{n}^{\circ}$ 2001-260 du 27 mars 2001 modifiant le code de l'urbanisme et le code de l'expropriation pour cause d'utilité publique et relatif aux documents d'urbanisme (JORF n ${ }^{\circ} 74$ du 28 mars 2001, p. 4836, texte $\mathrm{n}^{\circ}$ 92), faisant suite à la loi 2000-1208 du 13 décembre 2000 relative à la solidarité et au renouvellement urbains (JORF $\mathrm{n}^{\circ} 289$ du 14 décembre 2000, p. 19777, texte $\mathrm{n}^{\circ} 2$ )

7. Le classement en zone $\mathrm{N}$ n'empêchant pas l'exercice d'une activité agricole : Rép. min. $\mathrm{n}^{\circ}$ 56480, JOAN Q, 26 avril 2005, p. 4294.

8. Il importe de distinguer la zone A, qui relève du Code de l'urbanisme, de la « zone agricole protégée »(ZAP), qui relève essentiellement du Code rural. Délimitée par arrêté préfectoral au terme d'une procédure fixée par ce dernier, elle concerne les « zones agricoles dont la préservation présente un intérêt général en raison soit de la qualité de leur production, soit de leur situation géographique, soit de leur qualité agronomique »(C. rur., art. L. 112-2, al. 1 ; v. art. R. 112-1-4 et s.) et créé une servitude d'utilité publique annexée au PLU (C. rur., art. L. 112-2, al. 4) qui limite les changements d'affectation ou de mode d'occupation du sol (C. rur., art. L. 112-2, al. 2). Sa portée est tout de même limitée car ne sont pas concernés les changements de mode d'occupation qui relèvent d'une autorisation au titre du Code de l'urbanisme et lorsque le terrain est situé à l'intérieur d'un plan d'occupation des sols rendu public ou approuvé ou d'un document d'urbanisme en tenant lieu (C. rur., art. L. 112-2, al. 3). V. par exemple CAA 
Douai, 25 janvier 2007, ${ }^{\circ}$ 06DA01289. V. aussi H. Delesalle,

«Quelques précisions sur un instrument méconnu, les zones agricoles protégées », AJDA, n 36, 2015, p. 2052-2055.

9. Plus largement, sur la protection des terres agricoles en milieu périurbain : Ch. Debouy, «Les outils de protection des terres agricoles en milieu périurbain », AJDA, n 25, 2011, p. 1419-1426.

10. Cela n'est pas sans rappeler la carte communale qui « délimite les secteurs où les constructions sont autorisées et les secteurs où les constructions ne sont pas admises [sauf exception] » (C. urb., art. L. 161-4), ou le Règlement national d'urbanisme qui distingue les espaces urbanisés, dans lesquels les constructions peuvent être autorisées, de ceux qui ne le sont pas, les constructions ne pouvant là, sauf exception, être autorisées (règle de la constructibilité limitée : C. urb., art. L. 111-3 et s.).

11. CE, 18 novembre 1998, Mme Klein, $\mathrm{n}^{\circ} 164090$.

12. Ainsi, pour le juge administratif, le fait que des travaux ne sont pas soumis à une procédure spécifique ne fait pas obstacle à l'opposabilité des dispositions figurant dans le règlement $(\mathrm{CE}, 26$ novembre 2010, Ministre d'état, ministre de l'Écologie, du Développement durable, et de l'Aménagement du territoire, $\mathrm{n}^{\circ} 320871$ : BJDU 2011, $\mathrm{n}^{\circ}$ 2, p. 115, concl. Guyomar). Dans le même sens, une interdiction de construire ne saurait être opposée aux autorisations d'urbanisme si elle ne figure pas dans le règlement (CE, 26 mai 2010, Dos Santos, $n^{\circ} 320780$ : BJDU 2010, n 3, p. 193, concl. Geffray ; Constr.-Urb. 2010, $\mathrm{n}^{\circ}$ 9, p. 19, note Godfrin ; Envir. 2010, n 8-9, p. 46, note Gillig ; RDI 2010, $\mathrm{n}^{\circ}$ 7, p. 404, note SolerCouteaux ; RJE 2011, n 3,

p. 446, chron. Perez).

13. Pour la haute juridiction administrative, la qualification d' " équipement public » peut être retenue lorsqu'un projet « présente un intérêt public tiré de sa contribution à la satisfaction d'un besoin collectif par la production d'électricité vendue au public » : CE, 13 juillet 2012, Société EDP Renewables France, $\mathrm{n}^{\circ} 343306$.

14. Rép. min. $\mathrm{n}^{\circ} 77107$, JOAN Q, 17 janvier 2006, p. 296.

15.Rép. min. $\mathrm{n}^{\circ}$ 17006, JOAN Q, 24 juin 2008, p. 5399. V. aussi M. Boiron Bertrand, « Agri- culture, photovoltaïsme et urbanisme : points de convergence », RDI, n² 2, 2020, p. 66.

16.CE, 12 juillet 2019, $\mathrm{n}^{\circ} 422542$ : AJDA 2019, $\mathrm{n}^{\circ}$ 40, p. 2397, concl. Dutheillet de Lamothe ; Constr.Urb. 2019, n 9, p. 19, note Couton ; Dr. rur. 2019, n 478, p. 46, note Gueydan ; RDI 2019, n 9, p. 473, note Soler-Couteaux.

17. Sur ce point le juge exerce un contrôle normal et n'hésite pas à annuler les délibérations approuvant le SCoT en cas de justification insuffisante des choix d'objectifs chiffrés de limitation de la consommation foncière : par ex., CAA Bordeaux, 28 décembre 2017, Syndicat mixte du bassin d'Arcachon et du Val de l'Eyre, $\mathrm{n}^{\circ}$ 15BX02851 : RDI 2018, n 4, p. 240, note Soler-Couteaux.

18. Il est à noter que cette précision relative aux enjeux propres à chaque secteur est issue de la loi $\mathrm{n}^{\circ}$ 2014-1170 du 13 octobre 2014 d'avenir pour l'agriculture, l'alimentation et la forêt (JORF n $^{\circ} 0238 \mathrm{du} 14$ octobre 2014, p. 16601, texte $n^{\circ} 1$ ) qui avait enrichi l'ancien art. L. 122-1-5 du code de l'urbanisme qui traitait du document d'orientation et d'objectifs. Cette précision est intervenue pour améliorer la maîtrise de l'urbanisation et réduire la consommation des espaces agricoles, car, ainsi que le relevait l'étude d'impact de la loi, les SCoT étaient « souvent peu précis quant à la localisation des objectifs de limitation de consommation d'espace, ce qui rend[ait] leur opposabilité aux PLU non opérationnelle " (Étude d'impact du projet de loi d'avenir pour l'agriculture, l'alimentation et la forêt, 12 novembre 2013, p. 5657).

19. CE, 18 décembre 2017, Regroupement des organismes de sauvegarde de l'Oise, $\mathrm{n}^{\circ} 395216$ : AJDA 2018, n 23, p. 1348, note Tremeau ; BJDU 2018, n 2, p. 94, concl. Burguburu, note de Lesquen ; Constr.Urb. 2018, n $^{\circ}$ 2, p. 11, note Santoni ; JCP A 2018, n 22, p. 14, chron. Vandermeeren ; RDI 2018, ${ }^{\circ}$ 2, p. 
125 , note Strebler.

20. CE, 5 mars 2014, Commune de La Possession, $\mathrm{n}^{\circ} 363871$ : RJE 2015, $\mathrm{n}^{\circ}$ 1, p. 129, note Peyen.

21. Ch. Cans, «Le principe de conciliation : vers un contrôle de la «durabilité »? », in Terres du droit. Mélanges en 1'honneur de Yves Jégouzo, Dalloz, 2009, p. 547-572. V. encore récemment sur cette nécessité de conciliation : Cons. Constit., 31 janvier 2020, Union des industries de la protection des plantes, $\mathrm{n}^{\circ}$ 2019-823 QPC : Dr. adm. 2020, n $^{\circ}$ 4, comm. 17, note Fonbaustier ; Énergie-Env.-Infr. 2020, $\mathrm{n}^{\circ} 3$, p. 43, note Torre-Schaub ; JCP G 2020, n 10, p. 466, note Aguila et Rollini.

22. J.-F. Rouhaud, « La protection des espaces ruraux par les documents d'urbanisme : quelle efficacité pour l'exercice des activités agricoles ? » Dr. rur., n 399, janvier 2012, p. 26-32. À propos de la double nature, juridique et politique, du PLU, et son impact sur sa contribu- tion à la protection de l'environnement : B. Barraud, « Le plan local d'urbanisme, outil de protection de l'environnement ?», Dr. adm., $\mathrm{n}^{\circ}$ 2, fév. 2018, p. 20-25.

23. V. ici aussi CAA Lyon, 2 avril 2020, n 19LY00811.

24. En revanche, les collectivités publiques devant notamment assurer « la prévention des risques naturels prévisibles, des risques miniers, des risques technologiques, des pollutions et des nuisances de toute nature » (C. urb., art. L. 101-2, 5), le règlement du PLU peut tout à fait fixer des restrictions et des interdictions pour l'implantation de ces installations (C. urb., art. L. 152-1), et ce, même s'il existe une législation spécifique en la matière (pour un exemple en zone NC : CE, 12 février 1997, Société SOCCOIM, $\mathrm{n}^{\circ} 156895$ : RJE 1997, $\mathrm{n}^{\circ} 3$, p. 451, note Monédiaire).

25. J.-B. Auby, « Réflexions sur les caractères de la règle d'urbanisme », RDI, n 1, 1995, p. 39-50.

26. Rép. min. ${ }^{\circ} 79435$, JOAN Q, 17 août 2010, p. 9025.

27. Il convient de relever qu'il a déjà été admis qu'un POS puisse soumettre à des conditions restrictives les épandages de fumier et de lisier et d'autres dépôts sur des parcelles classées en zones NC. Néanmoins, il était moins question de limiter l'activité agricole ou de sous- traire les parcelles concernées à leur vocation agricole que d'encadrer ces pratiques qui étaient possiblement des sources de nuisances pour les zones urbaines situées à proximité immédiate : CE, 4 décembre 1995, Chambre d'agriculture de la Mayenne, $\mathrm{n}^{\circ} 128057$ : RJE 1996, $\mathrm{n}^{\circ}$ 3, p. 353, note Monédiaire.

28. CE, 1er décembre 2006, Société GFLBI, n 296543 : BJDU 2007, n 3, p. 183, concl. Mitjavile, note Bonichot ; Constr.-Urb. 2007, n 1, p. 22, note Godfrin ; LPA 2007, n 234, p. 8, note Fouilleul ; RDI 2007, n 2, p. 191, note Soler-Couteaux. V. aussi C. urb., art. L. 152-1.

29. Le juge administratif estime qu'il lui appartient « de rechercher, dans le cadre d'une analyse globale le conduisant à se placer à l'échelle du territoire couvert par le document d'urbanisme, si le règlement ne contrarie pas les orientations générales et objectifs que les auteurs du document ont définis dans le projet d'aménagement et de développement durables, compte tenu de leur degré de précision » : CE, 30 mai 2018, Commune de Sète, $n^{\circ} 408068$ : BJDU 2018, n 6, p. 386, note Burguburu ; JCP A 2018, $\mathrm{n}^{\circ}$ 48, p. 14, note Vandermeeren ; LPA 2018, $\mathrm{n}^{\circ}$ 177, p. 5, note Niel et Morin ; Constr.-Urb. 2018, $\mathrm{n}^{\circ} 10$, p. 14, note Santoni ; RDI 2018, ${ }^{\circ} 7$, p. 403, note Soler-Couteaux.

30. CAA Lyon, 28 février 2012, $\mathrm{n}^{\circ}$ 10LY02300.

31. CAA Bordeaux, 23 avril 2013, n 11BX00640.

32. V. M. Desrousseaux et L. Stahl, «L'appréhension de l'agriculture urbaine par le droit français », Géocarrefour, vol. 89-1-2, 2014, p. 65-73, spéc. p. 67.

33. Il suffit qu'ils soient « cultivés », c'est-à-dire qu'il peut s'agir de jardins d'agrément, de potagers ou de verger (CE, 12 juillet 1993, Communauté urbaine de Strasbourg, $\mathrm{n}^{\circ}$ 115247, 115253, 115447). A contrario, le dispositif ne peut s'appliquer pour des parcelles qui « n'ont pas fait l'objet de cultures dans le passé » et qui « sont toujours à l'état de friches » (CAA Versailles, 6 avril 2006, ${ }^{\circ}$ 04VE02945). 

nisation « des terrains à vocation agricole sans privilégier, au préalable, la densification des secteurs déjà urbanisés de la commune » (TA Melun, 23 février 2018, $\mathrm{n}^{\circ}$ 1608988).

35. V. sur ce point J. Bétaille, « Le décloisonnement du principe de précaution, un effet de sa constitutionnalisation », Dr. env., $\mathrm{n}^{\circ} 182,2010$, p. 278-279.

36. Y. Jegouzo, « Le droit de l'urbanisme, le bateau ivre ? », in Florilèges du droit public. Recueil de mélanges en l'honneur de Jean-Pierre Boivin, La Mémoire du Droit, 2012, p. 517-530.

37. CE, 20 novembre 2000, Commune de Guichen, ${ }^{\circ} 206262$.

38. CE, 6 juin 2007, Communauté urbaine Marseille-Provence-Métropole, $\mathrm{n}^{\circ} 266656$ : BJDU 2007, $\mathrm{n}^{\circ}$ 2, p. 113, concl. Verclytte, note Bonichot ; Constr.-Urb. 2007, n 7-8, p. 50, note Godfrin. Il est permis de douter que de tels motifs puissent valoir pour les zones A, qui n'interviennent pas, contrairement aux zones NC, pour protéger « la richesse du sol ou du sous-sol ». Reste qu'il est possible de l'envisager sous l'angle du potentiel agronomique, biologique ou économique des terres agricoles.

39. Sur les rapports entre documents d'urbanisme et environnement : M. Prieur (en collab.), Droit de l'environnement, op. cit., p. 1085 et s. (spéc. p. 1091 et s. à propos du PLU).

40. À propos d'une zone NC: CAA Versailles, 29 décembre 2011, Commune d'Épinay-Champlâtreux, n 10VE00269; CE, 12 février 2014, Commune d'Épinay-Champlâtreux, n $^{\circ} 357215$ : AJDA $2014, n^{\circ} 23$, p. 1338, note Pouthier.

41. V. la contribution de C. Gueydan dans ce dossier.

42. CAA Marseille, 20 juin 2017, $\mathrm{n}^{\circ}$ 16MA01191.

43. Ph. Billet, « Droit de préemption et protection de l'environnement », Constr.-Urb., $\mathrm{n}^{\circ}$ 9, sept. 2009, p. 13-19 ; M. Saint-Supery et D. Kacete, « Le droit de préemption dans les espaces naturels sensibles », Constr.-Urb., n 12, déc. 2018, p. 7-12.

44. À propos des zones NC des POS : CE, 16 juin 1995, Préfet des Yvelines, ${ }^{\circ} 140022$ : RJE 1997, $\mathrm{n}^{\circ}$ 2, p. 173, note Périnet-Marquet ; CE, 3 juillet 1998, Préfet des Yvelines, $\mathrm{n}^{\circ} 132250$.

45. Loi $\quad$ L 2016-1087 du 8 août 2016 pour la reconquête de la biodiversité, de la nature et des paysages, JORF n ${ }^{\circ} 0184$ du 9 août 2016 , texte $n^{\circ} 2$.

46. À titre de comparaison, à propos des schémas régionaux de cohérence écologique (qui ont maintenant intégré le schéma régional d'aménagement, de développement durable et d'égalité des territoires, excepté en Ile-de-France), le juge administratif vérifie que le diagnostic du territoire régional qu'il comprend porte aussi sur les interactions entre la biodiversité et les activités humaines, sans exercer un contrôle plus approfondi en substance : CE, 30 juillet 2014, Fédération départementale des chasseurs du Pas-de-Calais, ${ }^{\circ} 369148$.

47. J. Makowiak, « Les espaces ruraux et naturels à l'épreuve du développement durable », AJDA, $\mathrm{n}^{\circ} 23,2005$, p. 1268-1273.

48. D'une autre perspective, sur ce thème : P. Soler-Couteaux, « Le droit de l'urbanisme permissif », RDI, $\mathrm{n}^{\circ}$ 1, 2020, p. 8-14.

49. H. Périnet-Marquet, « Propos conclusifs », RDI, n 1, 2020, p. 47-52.

50. G. Roux, « La notion de construction « liée et nécessaire à l'activité agricole », AJDA, n 18, 2018, p. 1020-1025.

51. À propos des annexes : D. Gillig, « La possibilité de réaliser des constructions annexes en zones naturelles et agricoles », Constr.-Urb., n 12, déc. 2018, p. 3-4.

52. P. Soler-Couteaux et E. Carpentier, Droit de 1'urbanisme, 7e éd., Dalloz, 2019, p. 250 et s. Sur le régime juridique de la zone NC et, en particulier, les questions de constructibilité, v. L. Yousry, «Les zones agricoles dans les plans d'occupation des sols et le juge adminis- tratif », Dr. rur., $\mathrm{n}^{\circ} 179,1990$, p. 38 et $\mathrm{s}$. 
53. Loi $\mathrm{n}^{\circ} 2003-590$ du 2 juillet 2003 urbanisme et habitat, JORF $\mathrm{n}^{\circ} 152$ du 3 juillet 2003, p. 11176, texte $n^{\circ} 1$.

54. Loi $\mathrm{n}^{\circ} 2010-788$ du 12 juillet 2010 portant engagement national pour l'environnement, JORF $\mathrm{n}^{\circ}$ 0160 du 13 juillet 2010, p. 12905, texte $\mathrm{n}^{\circ} 1$; D. Gillig, « La jurisprudence Commune de Châteauneufdu-Rhone est morte ! Vive le pastillage en zone agricole ! », JCP A, n 26, 28 juin 2010, act. 533.

55. Loi n $\mathrm{n}^{\circ}$ 2014-366 du 24 mars 2014 pour l'accès au logement et un urbanisme rénové, JORF $\mathrm{n}^{\circ} 0072$ du 26 mars 2014, p. 5809, texte $\mathrm{n}^{\circ} 1$. Il convient de noter que si cette loi faisait réfé- rence à la possibilité d'une extension "limitée », cet adjectif a disparu par la suite, la même année, avec la loi n 2014-1170 du 13 octobre 2014 d'avenir pour l'agriculture, l'alimentation et la forêt (JORF n ${ }^{\circ} 0238 \mathrm{du} 14$ octobre 2014 , p. 16601 , texte $\mathrm{n}^{\circ} 1$ ).

56. Loi n $\quad$ 2015-990 du 6 août 2015 pour la croissance, l'activité et l'égalité des chances économiques, JORF n ${ }^{\circ} 0181$ du 7 août 2015, p. 13537, texte ${ }^{\circ} 1$.

57. Décret $n^{\circ}$ 2015-1783 du 28 décembre 2015 relatif à la partie réglementaire du livre Ier du code de l'urbanisme et à la modernisation du contenu du plan local d'urbanisme, JORF n ${ }^{\circ} 0301$ du 29 décembre 2015 , p. 24530 , texte $\mathrm{n}^{\circ} 78$.

58. Loi $\mathrm{n}^{\circ}$ 2018-1021 du 23 novembre 2018 portant évolution du logement, de l'aménagement et du numérique, JORF $\mathrm{n}^{\circ} 0272$ du 24 novembre 2018 , texte $\mathrm{n}^{\circ} 1$.

59. V. par ex. à propos d'une centrale photovoltaïque : CAA Marseille, 11 décembre 2018, Société Centrale Solaire Orion 6, $\mathrm{n}^{\circ}$ 17MA045

60. CE, 8 février 2017, Ministre du logement et de l'habitat durable c. Société Photosol, n 395464 : BJDU 2017, n 3, p. 156, concl. Decout-Paolini, note De Lesquen ; Dr. rur. 2017, ${ }^{\circ}$ 453, p. 32, note Lochouarn; JCP A 2017, n 29, p. 17, note Vandermeeren ; RDI 2017, ${ }^{\circ}{ }^{\circ}$, p. 246, note Decout-Paolini.

61. À propos des secteurs de taille et de capacité d'accueil limitées, en zone ND cependant : CAA Nantes, 17 janvier 2014, Association pour la protection et la promotion du château d'Ancenis, $\mathrm{n}^{\circ}$ 12 NT01588.

62. V. l'éclairant arrêt de la CAA de Nantes statuant sur renvoi du Conseil d'État (v. arrêt Société EDP Renewables France, op. cit.) : CAA Nantes, 15 novembre 2013, Société EDP Renewables France, $\mathrm{n}^{\circ} 12 \mathrm{NT} 02171$.

63. V. par ex. à propos de la zone littorale : T. Gilliocq, « La constructibilité en zone agricole littorale », JCP A, 13 février 2017, n 6, p. 31-41 ; R. Leroy, « La loi littoral et les activités agricoles », RJE, nº spéc., 2012, p. 137-148.

64. B. Grimonprez, «Agriculture et zones humides : un droit entre deux eaux », Dr. rur., n 393 , mai 2011, p. 15-21.

65. J.-M. Février, « La loi sur le développement des territoires ruraux et Natura 2000 », Envir., ${ }^{\circ}$ 5, mai 2005, p. 11-13.

66. R. Romi, « La loi sur le développement des territoires ruraux : le droit de l'environnement saisi par le ruralisme ou le ruralisme saisi par le droit de l'environnement ? », RJE, n 4, 2005, p. 405-424 ; A. Charlez, « La loi sur le développement des territoires ruraux et le droit de l'environnement », Dr. rur., $\mathrm{n}^{\circ}$ 338, décembre 2005, p. 24-28.

67. Y. Jégouzo, «Les espaces partiellement affectés à la protection de la nature », Dr. rur., $\mathrm{n}^{\circ} 75$, avril 1979 , p. 123 et s.

68. J.-M. Auby, «Quelques réflexions sur l'état du droit de l'urbanisme», in Mélanges en l'hon- neur de Henri Jacquot, PUO, 2006, p. 31-40 ; I. Savarit-Bourgeois, « Remarques sur quelques dérives du droit de l'urbanisme », in Le droit administratif: permanences et convergences, Mélanges en l'honneur de JeanFrançois Lachaume, Dalloz, 2007, p. 939-959. 